\title{
The intrinsic Baldwin effect in broad Balmer lines of six long-term monitored AGNs
}

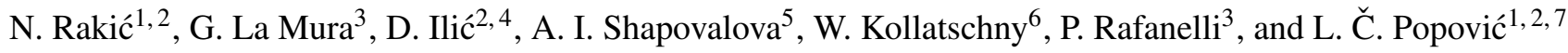 \\ ${ }^{1}$ Faculty of Science, University of Banjaluka, Mladena Stojanovića 2, 78000 Banjaluka, Republic of Srpska, Bosnia and Herzegovina \\ e-mail: nemanja.rakic@unibl.rs \\ 2 Department of Astronomy, Faculty of Mathematics, University of Belgrade, Studentski Trg 16, 11000 Belgrade, Serbia \\ 3 Department of Physics and Astronomy, University of Padova, vicolo dell'Osservatorio 3, 35122 Padova, Italy \\ 4 Isaac Newton Institute of Chile, Yugoslavia Branch, 11060 Belgrade, Serbia \\ 5 Special Astrophysical Observatory of the Russian Academy of Science, Nizhnij Arkhyz, 369167 Karachaevo-Cherkesia, Russia \\ 6 Institut fur Astrophysik, Universitat Gottingen, Friedrich-Hund Platz 1, 37077 Göttingen, Germany \\ 7 Astronomical Observatory, Volgina 7, 11060 Belgrade, Serbia
}

Received 18 November 2016 / Accepted 17 March 2017

\begin{abstract}
We investigate the intrinsic Baldwin effect (Beff) of the broad $\mathrm{H} \alpha$ and $\mathrm{H} \beta$ emission lines for six Type 1 active galactic nuclei (AGNs) with different broad line characteristics: two Seyfert 1 (NGC 4151 and NGC 5548), two AGNs with double-peaked broad line profiles (3C 390.3 and Arp 102B), one narrow line Seyfert 1 (Ark 564), and one high-luminosity quasar with highly red asymmetric broad line profiles (E1821+643). We find that a significant intrinsic Beff was present in all Type 1 AGNs in our sample. Moreover, we do not see strong differences in intrinsic Beff slopes in different types of AGNs, which probably have different physical properties such as inclination, broad line region (BLR) geometry, or accretion rate. Additionally, we find that the intrinsic Beff was not connected with the global Beff, which, instead, could not be detected in the broad $\mathrm{H} \alpha$ or $\mathrm{H} \beta$ emission lines. In the case of NGC 4151, the detected variation of the Beff slope could be due to the change in the site of line formation in the BLR. Finally, the intrinsic Beff might be caused by the additional optical continuum component that is not part of the ionization continuum.
\end{abstract}

Key words. galaxies: active - galaxies: Seyfert - quasars: emission lines - quasars: general

\section{Introduction}

Correlations between the continuum and line emission of active galactic nuclei (AGNs) are a valuable probe of the physics and structure of the central engine and its close environment. For a sample of objects, Baldwin (1977) found that the equivalent width (EW) of the C IV $\lambda 1549$ emission line was anti-correlated with the luminosity of the underlying continuum. This property, named "the Baldwin effect (Beff)" by Carswell \& Smith (1978), was detected in almost all UV and optical broad emission lines (see, e.g., Dietrich et al. 2002; Dong et al. 2009; Kovačević et al. 2010; Shemmer \& Lieber 2015), in the narrow lines (see Shields 2007 , for a review) though with different strengths, and also in the X-ray spectra of AGNs (see, e.g., Jiang et al. 2006, 2007; Shu et al. 2012; Ricci et al. 2013).

Today, two different types of Baldwin effect are present in the literature:

1. global or ensemble Baldwin effect - anti-correlation between the EW of the emission line and the underlying continuum luminosity of single-epoch observations of a large number of AGNs.

2. intrinsic Baldwin effect - anti-correlation between the EW of the emission line and the underlying continuum of individual variable AGNs (Pogge \& Peterson 1992).

The existence of an intrinsic correlation and its relation with the global effect provides important clues about how the broad emission lines are produced, and it is therefore important in order to better constrain the nature of the broad line region (BLR).
For this reason, the Beff has been investigated in several papers and different mechanisms have been proposed as possible interpretations. In spite of the numerous efforts to clarify its nature, the question of the physical meaning of the Beff has long been a matter of debate (see, e.g., Boroson \& Green 1992; Netzer et al. 1992; Zheng et al. 1992; Zheng \& Malkan 1993; Green 1996, 1998; Korista et al. 1998; Warner et al. 2003; Bachev et al. 2004; Baskin \& Laor 2004; Xu et al. 2008; Popović \& Kovačević 2011; Bian et al. 2012).

The main explanations that have been suggested are as follows:

(i) a luminosity dependence of the ionization parameter $U$ and an anti-correlation between the continuum luminosity and the BLR covering factor (Mushotzky \& Ferland 1984);

(ii) a geometrical effect due to the combination of an inclination dependent anisotropic continuum with a more isotropic line emission (Netzer 1985);

(iii) a different variability pattern in the thermal and nonthermal components of the continuum, leading to changes in the spectral energy distribution (SED) of the ionizing photons, which affect the broad line intensities independently of the state of the continuum close to the line (Kinney et al. 1990);

(iv) a multiple component nature of the broad emission lines, with part of the line flux not directly controlled by the continuum luminosity (Pogge \& Peterson 1992; Shapovalova et al. 2008);

(v) a general trend of softer continuum shapes and enhanced metallicities at higher luminosities (Dietrich et al. 2002); 
(vi) a different Eddington ratio (see Calvani et al. 2004; Baskin \& Laor 2004; Bachev et al. 2004; Marziani et al. 2008; Dong et al. 2009; Bian et al. 2012) or mass of the supermassive black hole (SMBH) Xu et al. (2008).

Different combinations of the fundamental effects listed above can be used to explain both the intrinsic and global Beff.

In the case of the global Beff, it has been established that the higher ionization lines show steeper slopes in the EW versus $L$ plot (see Fig. 9 in Dietrich et al. 2002). Some investigations have revealed that the Balmer lines show no global Beff (see, e.g., Kovačević et al. 2010; Popović \& Kovačević 2011) or even a weak inverse global Beff (positive correlation between a line and corresponding continuum Croom et al. 2002; Greene \& Ho 2005). Zamorani et al. (1992) combined geometrical effects with the luminosity dependent ionization parameter $U$ to explain the Beff of high- and low-ionization lines, whereas Zheng \& Malkan (1993) suggested that the most luminous objects could produce relatively softer continua, resulting in weaker high-ionization lines. In general, the possibility that different SMBH masses and accretion rates, together with changes in the gas metallicity, could be combined to provide an interpretation of the global Beff in the framework of cosmological evolution is often suggested (Korista 1999; Green et al. 2001; Shang et al. 2003).

On the other hand, the intrinsic Beff has been studied in several papers, but only for strongly variable AGNs. Kinney et al. (1990) observed a set of seven objects observed with the IUE satellite for more than 15 epochs, and found the presence of the intrinsic Beff in the UV line CIV and Ly $\alpha$. Gilbert \& Peterson (2003) showed that the intrinsic Beff is present in the broad $\mathrm{H} \beta$ line of NGC 5548. They considered the light travel times from the continuum source to the line emitting region in NGC 5548 and found that an intrinsic Beff held even when comparing the broad line EWs with the continuum that actually affected the gas at the time of the line emission. Goad et al. (2004) showed that photoionization models predicted a time variable response of emission lines from an extended BLR that was entirely consistent with $\mathrm{H} \beta$ line observations. Interestingly, variations in the slope of the intrinsic Beff have been found so far for the C IV line in NGC 4151 (Kong et al. 2006) and Fairall 9 (Wamsteker \& Colina 1986; Osmer \& Shields 1999), and for $\mathrm{H} \beta$ in NGC 5548 (Goad et al. 2004). This shows that a nonconstant slope may be common for AGNs. Moreover, the intrinsic Beff has a steeper slope than global Beff (Kinney et al. 1990; Pogge \& Peterson 1992). An additional detail seen in the intrinsic Beff is that the slope in $\log (E W)$ versus $\log L$ relation steepens when the source is becoming more luminous (see, e.g., Kong et al. 2006). This trend of the slope steepening for higher continuum luminosities is also observed in the global Beff (Osmer \& Shields 1999; Baskin \& Laor 2004). However, the global and intrinsic Baldwin effects probably have completely different physical origins, thus they are unlikely to be connected. For the intrinsic Beff, several authors have advocated the idea that it could be explained by the different phases in the activity of an accretion powered source (Wandel 1999a,b; Kong et al. 2006).

The aim of this work is to investigate the intrinsic broad line Beff in Type 1 AGNs with different characteristics in order to explore the effect of the BLR orientation and geometry on the intrinsic Beff and to discuss the lack of global Beff in the broad Balmer lines. Therefore, we combine observations of six long-term monitored objects: two typical Seyfert 1 galaxies (NGC 4151 and NGC 5548), two AGNs with double-peaked broad lines (3C 390.3 and Arp 102B), one narrow line Seyfert 1
- NLSy1 galaxy (Ark 564), and one high-luminosity quasar with highly red asymmetric broad line profiles (E1821+643). We explore the intrinsic Beff in the broad $\mathrm{H} \alpha$ and $\mathrm{H} \beta$ emission lines, and the $\mathrm{H} \alpha$ and $\mathrm{H} \beta$ ratio as a function of the continuum flux. We discuss the intrinsic Beff together with the global Beff. Finally, we speculate on the physical background of the intrinsic Beff.

The paper is organized as follows. In Sect. 2 we describe the sources of used data and the method of analysis. Our results and the discussion are presented in Sect. 3, and in Sect. 4 we discuss some possible physical processes that cause the intrinsic Beff. Finally, in Sect. 5 we outline our conclusions.

\section{Data and method of analysis}

To construct a uniform and consistent data set for all AGNs, we used the observational data with already measured line and continuum fluxes from our monitoring campaigns (see Ilić et al. 2015 , and references therein for more details). The sample contains six long-term monitored AGNs with different spectral characteristics and subclassifications:

1. Seyfert 1 AGNs NGC 5548 and NGC 4151, which showed strong variability in the broad spectral lines (full width at half maximum $(F W H M) \sim 5000-7000 \mathrm{kms}^{-1}$ ) and continuum flux (see, e.g., Shapovalova et al. 2004, 2008, 2010b). Moreover, their broad line profiles exhibit noticeable change in line shape and intensity, resulting in periods when these AGNs almost show the characteristics of Seyfert 2 (very weak broad lines; see Shapovalova et al. 2004, 2008, 2010b);

2. Double-peaked broad line AGNs 3C 390.3 and Arp 102B, whose broad Balmer lines show two distinguished peaks with very large overall width $\left(F W H M \sim 10000 \mathrm{~km} \mathrm{~s}^{-1}\right)$. This type of objects are called double-peaked line (DPL) AGNs. These lines are supposed to be emitted from an accretion disk (Chen et al. 1989). 3C 390.3, which is a radio loud $\mathrm{AGN}$, seems to have higher variability in the spectra (Shapovalova et al. 2010a) than Arp 102B (Shapovalova et al. 2013);

3. Narrow line Seyfert 1 (NLSy1) AGN Ark 564, which has a strong Fe II emission and somewhat narrower broad lines $\left(F W H M \sim 2000 \mathrm{~km} \mathrm{~s}^{-1}\right.$ ) with a prominent narrow component. The variability of this AGN is weak, but there are occasional flare-like outbursts (see Shapovalova et al. 2012);

4. High-luminosity quasar E1821+643, a low-redshift $(z=0.297)$ radio-quiet quasar. Its broad emission lines $\left(F W H M>5000 \mathrm{~km} \mathrm{~s}^{-1}\right)$ are redshifted by $\sim 1000 \mathrm{~km} \mathrm{~s}^{-1}$ and have strongly asymmetric profiles in the red part. These effects make this object a candidate for either a supermassive binary black hole (SMBBH) or a recoiling black hole after SMBH collision (Robinson et al. 2010; Shapovalova et al. 2016). It is a weakly variable quasar, but some flare-like outbursts have been detected (see Shapovalova et al. 2016).

For the comparison of the intrinsic Beff in our sample with the global Beff trend, we used the spectra of 21416 Type 1 AGNs taken from the Sloan Digital Sky Survey (SDSS) database studied by Shen et al. (2011), combined with a sample of 90 lowluminosity objects, also from the SDSS database, analyzed in detail by La Mura et al. (2007) in order to cover a wider luminosity range. Furthermore, from Shen et al. (2011) we extracted the subsample of 4800 broad line AGNs to study the $\mathrm{H} \alpha / \mathrm{H} \beta$ ratio.

We adopt the cosmological parameters used in Shen et al. (2011), $H_{0}=70 \mathrm{~km} \mathrm{~s}^{-1} \mathrm{Mpc}, \Omega_{\text {matter }}=0.30$, and $\Omega_{\text {vacuum }}=0.70$ when calculating the luminosity and luminosity distance. 


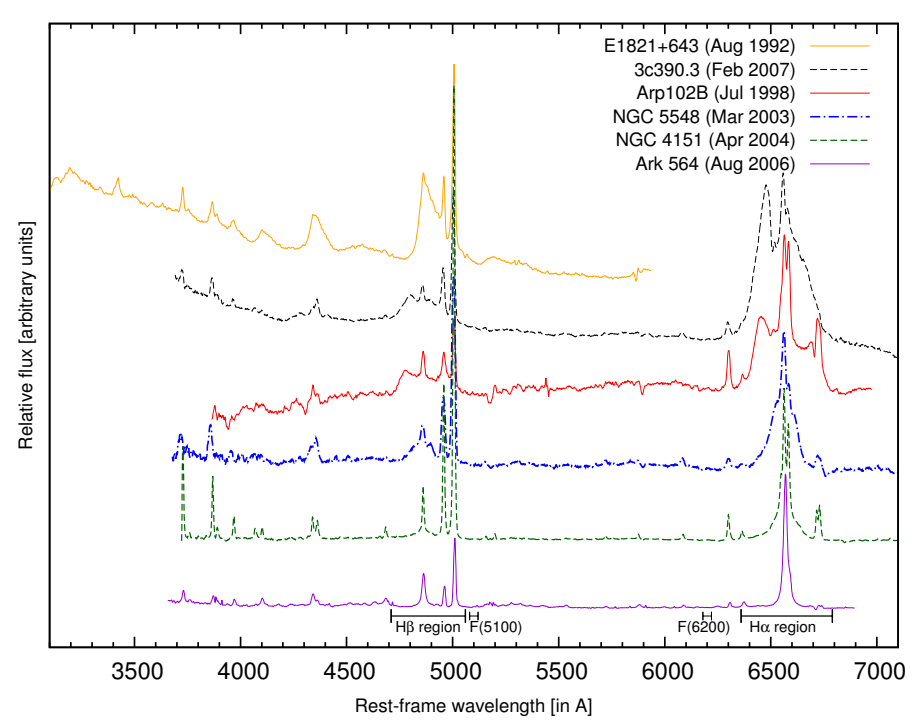

Fig. 1. Spectra of six studied objects (as listed in top right corner, object name and observation epoch are given). Intensity is given in arbitrary units shown for comparison. The measured continuum and line extraction bands are marked below the first spectrum.

\subsection{Data reduction}

Optical spectral data used in the investigation of the intrinsic Beff are taken from the published long-term monitoring campaigns described in several papers (see Shapovalova et al. 2004, 2008, 2010a, 2012, 2013, 2016), where a detailed description of the data reduction and flux measurements was given and therefore is not repeated here. We emphasize that all spectral data were reduced and analyzed using the same method in order to construct a uniform data set in a consistent way. We list below only some basic information. The spectra were obtained with the following instruments: (i) the $6 \mathrm{~m}$ and $1 \mathrm{~m}$ telescopes of the Special Astrophysical Observatory (SAO), Russia; (ii) the $2.1 \mathrm{~m}$ telescope of the Guillermo Haro Astrophysical Observatory (GHAO) at Cananea, Sonora, Mexico; (iii) the $2.1 \mathrm{~m}$ telescope of the Observatorio Astronómico Nacional at San Pedro Martir (OANSPM), Baja California, Mexico; and (iv) the $3.5 \mathrm{~m}$ and $2.2 \mathrm{~m}$ telescopes of the Calar Alto Observatory (CA), Almería, Spain. The typical properties of the observed spectra were the wavelength range 4000-7500 $\AA$, the spectral resolution $\Delta \lambda=5-15 \AA$, and the $\mathrm{S} / \mathrm{N}$ ratio $>50$ in the continuum near $\mathrm{H} \alpha$ and $\mathrm{H} \beta$. All spectra, taken with different telescopes, were flux-calibrated by scaling the overall spectra to have the same AGN narrow emission line fluxes. The typical line and continuum flux uncertainties are $\sim 3-5 \%$. The narrow line flux does not vary because the narrow line region (NLR) is ten to kpc scale, thus its variation in years is negligible (see, e.g., Shapovalova et al. 2004, 2008, 2010a, 2012, 2013, 2016; Runnoe et al. 2016). However, different aperture sizes will result in different narrow line fluxes, and consequently can affect the scaled continuum and broad line fluxes. Therefore, special attention is given to the correction of the continuum and broad line flux to aperture effects (see Peterson et al. 1995, 2002; Shemmer et al. 2001; Sergeev et al. 2002; Shapovalova et al. 2013, 2016), and we used the observed data that have been corrected for this effect. A summary of the observations and the data used is listed in Table 1. For illustration, in Fig. 1 we plot an example spectrum of each studied object in arbitrary units for comparison, shown in the order listed in the top right corner of Fig. 1.
Host-galaxy and narrow-line contribution. For the studies of the intrinsic Beff, it is important to correct the continuum fluxes for the host-galaxy contribution and line fluxes for the narrowline contribution.

For the host-galaxy contribution to the continuum flux at $5100 \AA$ and $6200 \AA$, we took the estimates for a given aperture size from the literature (see references in Table 2 for details of the procedures used). The estimated host-galaxy flux, the aperture size, and the references are listed in Table 2. For most objects, the aperture size used for our flux measurements is similar to the aperture size used to estimate the host-galaxy flux (see Tables 1 and 2), thus we simply subtracted the host-galaxy flux from the continuum flux. Only in the case of NGC 5548 did we first correct the continuum flux to the aperture of $5^{\prime \prime} \times 7.5^{\prime \prime}$ used by Peterson et al. $(1995,2002)$ for the host-galaxy contribution. For the correction of the continuum at $6200 \AA$ of Ark 564, we calculated the scaled value from the host-galaxy flux at $5100 \AA$ as that was only available in the literature (Shemmer et al. 2001).

The narrow-line contribution to the $\mathrm{H} \alpha$ and $\mathrm{H} \beta$ emission lines was already subtracted in the published data for NGC 4151, NGC 5548, 3C 390.3, and E1821+643 (Shapovalova et al. 2004, 2008, 2010a, 2016). For Arp 102B, we took the Shapovalova et al. (2013) estimated values: $5.46 \times 10^{-14} \mathrm{erg} \mathrm{cm}^{-2} \mathrm{~s}^{-1}$ for the narrow $\mathrm{H} \beta+[\mathrm{O}$ III $] \lambda \lambda 4959$, 5007 lines, and $13.52 \times 10^{-14} \mathrm{erg} \mathrm{cm}^{-2} \mathrm{~s}^{-1}$ for the narrow $\mathrm{H} \alpha+[\mathrm{NII}] \lambda \lambda 6548,6584+[\mathrm{S} \mathrm{II}] \lambda \lambda 6717,6731$ lines. For the case of Ark 564 we took the Shapovalova et al. (2012) estimates: $20 \%$ narrow-line contribution to the mean $\mathrm{H} \beta$ flux, and $30 \%$ to the mean $\mathrm{H} \alpha$ flux.

\subsection{Method of analysis}

The Beff can be expressed as a simple power law relation:

$E W_{\lambda} \propto L_{\lambda}^{\beta}$

where $E W_{\lambda}$ is the equivalent width of the emission line (in $\AA$ ), $L_{\lambda}$ is the corresponding specific continuum luminosity (in $\operatorname{erg~s}^{-1} \AA^{-1}$ ) at wavelength close to the emission line, and $\beta$ is the slope. Hence, in order to have a Beff, the value must be $\beta<0$.

As we investigate the intrinsic Beff of a specific object, the luminosity can be replaced with the flux. It is very important to calculate $\mathrm{EW}$ in the way that represents the response of an emission line to the continuum that actually stroke the BLR at the line emission time (see, e.g., Gilbert \& Peterson 2003; Goad \& Korista 2014). Thus, we took into account the time delays due to light travel time from the continuum source to the BLR for each object obtained from the reverberation mapping. For this we first interpolated the continuum and emission line light curves, and then calculated the EW using the line flux that is corrected for the time delay (listed in Table 1 as photometric BLR radius) with respect to the continuum flux. We define the equivalent width of the $\mathrm{H} \beta$ and $\mathrm{H} \alpha$ lines as follows:

$E W=\frac{F_{\text {line }}(\mathrm{JD}+\tau)}{F_{\text {cnt }}(\mathrm{JD})}$,

where $F_{\text {cnt }}(\mathrm{JD})$ represents the continuum flux for a certain epoch and $F_{\text {line }}(\mathrm{JD}+\tau)$ is the broad line flux at the epoch corrected for the time delay $\tau$. In those cases where the continuum close to the $\mathrm{H} \alpha$ line was not measured, we used the blue continuum close to the $\mathrm{H} \beta$ line (see Table 1 ).

Assuming that the data follow a relationship of the form

$F_{\text {line }}=k F_{\text {cnt }}^{\alpha}$, 
Table 1. Basic information about the observations of the used spectra.

\begin{tabular}{|c|c|c|c|c|c|c|c|c|}
\hline Object $^{a}$ & Type $^{b}$ & Period $^{c}$ & Telescope $^{d}$ & Aperture $^{e}$ & Line $^{f}$ & Continuum $^{g}$ & No. ${ }^{h}$ & Reference $^{i}$ \\
\hline \multirow[t]{2}{*}{ NGC 5548} & Seyfert 1 & 1996-2002 & SAO, GHAO, OAN-SPM & $4.2^{\prime \prime} \times 19.8^{\prime \prime}$ & $\mathrm{H} \alpha$ & $5100 \AA$ & 23 & 1 \\
\hline & & & & & $\mathrm{H} \beta$ & $5100 \AA$ & 81 & \\
\hline \multirow[t]{2}{*}{ NGC 4151} & Seyfert 1 & 1996-2006 & SAO, GHAO, OAN-SPM & $2.0^{\prime \prime} \times 6.0^{\prime \prime}$ & $\mathrm{H} \alpha$ & $5100 \AA$ & 137 & 2 \\
\hline & & & & & $\mathrm{H} \beta$ & $5100 \AA$ & 181 & \\
\hline 3C 390.3 & DPL & 1995-2007 & SAO, GHAO, OAN-SPM & $2.0^{\prime \prime} \times 6.0^{\prime \prime}$ & $\mathrm{H} \beta$ & $5100 \AA$ & 128 & 3 \\
\hline \multirow[t]{2}{*}{ Arp 102B } & DPL & 1987-2013 & SAO, GHAO, OAN-SPM, CA & $2.5^{\prime \prime} \times 6.0^{\prime \prime}$ & $\mathrm{H} \alpha$ & $6200 \AA$ & 88 & 4 \\
\hline & & & & & $\mathrm{H} \beta$ & $5100 \AA$ & 116 & \\
\hline \multirow[t]{2}{*}{ Ark 564} & NLSy1 & 1999-2010 & SAO, GHAO, OAN-SPM & $2.5^{\prime \prime} \times 6.0^{\prime \prime}$ & $\mathrm{H} \alpha$ & $6200 \AA$ & 50 & 5 \\
\hline & & & & & $\mathrm{H} \beta$ & $5100 \AA$ & 91 & \\
\hline E1821+643 & quasar & 1990-2014 & $\mathrm{SAO}, \mathrm{GHAO}, \mathrm{CA}$ & $2^{\prime \prime} \times 6.0^{\prime \prime}$ & $\mathrm{H} \beta$ & $5100 \AA$ & 127 & 6 \\
\hline
\end{tabular}

Notes. Columns: ${ }^{(a)}$ Object name; ${ }^{(b)}$ AGN type; ${ }^{(c)}$ Monitored period; ${ }^{(d)}$ Telescope used (SAO - $6 \mathrm{~m}$ and $1 \mathrm{~m}$ telescopes of Special Astrophysical Observatory; GHAO - $2.1 \mathrm{~m}$ telescope of Guillermo Haro Astrophysical Observatory; OAN-SPM - $2.1 \mathrm{~m}$ telescope of the Observatorio Astronómico Nacional at San Pedro Martir; and CA - $3.5 \mathrm{~m}$ and $2.2 \mathrm{~m}$ telescopes of Calar Alto); ${ }^{(e)}$ Projected spectrograph entrance apertures (slit width $\times$ slit length in arcsec); ${ }^{(f)}$ E mission line; ${ }^{(g)}$ Continuum; ${ }^{(h)}$ Number of spectra; ${ }^{(i)}$ Reference from which line and continuum fluxes are taken.

References. (1) Shapovalova et al. (2004); (2) Shapovalova et al. (2008); (3) Shapovalova et al. (2010a); (4) Shapovalova et al. (2013); (5) Shapovalova et al. (2012); (6) Shapovalova et al. (2016).

Table 2. Properties of the AGNs in our sample used in the paper.

\begin{tabular}{cccccccccc}
\hline \hline Object $^{a}$ & Aperture $^{b}$ & $\begin{array}{c}F_{\text {gal }}{ }^{c} \\
\text { blue/red }\end{array}$ & Reference $^{d}$ & $\begin{array}{c}F W H M^{e} \\
\mathrm{H} / / \mathrm{H} \alpha\end{array}$ & $\begin{array}{c}R_{\mathrm{BLR}}{ }^{f} \\
\mathrm{H} \beta / \mathrm{H} \alpha\end{array}$ & $F_{\max } / F_{\min }{ }^{g} \beta / \mathrm{H} \alpha$ & Reference $^{h}$ & $\begin{array}{c}\mathrm{JD}^{2}(\mathrm{div})^{i} \\
240000+\end{array}$ & $F_{\text {cnt }}(\mathrm{div})^{j}$ \\
\hline NGC 4151 & $2.0^{\prime \prime} \times 6.0^{\prime \prime}$ & 6.00 & 1 & $6110 / 4650$ & $5 / 6$ & $4.8 / 3.1$ & 1 & 51283 & 46.2 \\
NGC 5548 & $5.0^{\prime \prime} \times 7.5^{\prime \prime}$ & 3.06 & 2,3 & 6300 & $49 / 27$ & $4.9 / 3.5$ & 8 & 51702 & 4.41 \\
3C 390.3 & $2.0^{\prime \prime} \times 6.0^{\prime \prime}$ & 0.46 & 4 & $11900 / 11000$ & $96 / 120$ & $4.7 / 3.4$ & 9 & 52327 & 0.74 \\
Arp 102B & $2.5^{\prime \prime} \times 6.0^{\prime \prime}$ & $1.13 / 1.21$ & 5 & $15900 / 14300$ & $15 / 21$ & $3.0 / 2.4$ & 5 & 55367 & 0.38 \\
Ark 564 & $2.5^{\prime \prime} \times 6.0^{\prime \prime}$ & $2.40 / 0.99$ & 6 & $960 / 800$ & $4 / 5$ & $1.6 / 1.5$ & 10 & 53505 & 2.55 \\
E1821+643 & $2.0^{\prime \prime} \times 6.0^{\prime \prime}$ & 0.29 & 7 & 5610 & 120 & 1.4 & 7 & 53683 & 10.35 \\
\hline
\end{tabular}

Notes. Columns: ${ }^{(a)}$ Object name; ${ }^{(b)}$ Projected spectrograph entrance aperture for the host galaxy; ${ }^{(c)}$ Host-galaxy contribution in units of $10^{-15} \mathrm{erg} \mathrm{cm}^{-2} \mathrm{~s}^{-1} \AA^{-1}$; ${ }^{(d)}$ References from which the entrance aperture and $F_{\text {galaxy }}$ are taken; ${ }^{(e)}$ FWHM of the mean line profile in units of $\mathrm{km} \mathrm{s}^{-1} ;{ }^{(f)}$ Photometric BLR radius in light days; ${ }^{(g)}$ Maximum to minimum line flux ratio; ${ }^{(h)}$ References from which the FWHM, $R_{\mathrm{BLR}}$, and $F_{\max } / F_{\min }$ are taken; ${ }^{(i-j)}$ modified Julian date dividing period 1 and 2 of different levels of activity and the corresponding continuum flux at $5100 \AA$ in units of $10^{-15} \mathrm{erg} \mathrm{cm}^{-2} \mathrm{~s}^{-1} \AA^{-1}$.

References. (1) Shapovalova et al. (2008); (2,3) Peterson et al. (1995, 2002); (4) Sergeev et al. (2002); (5) Shapovalova et al. (2013); (6) Shemmer et al. (2001); (7) Shapovalova et al. (2016); (8) Shapovalova et al. (2004); (9) Shapovalova et al. (2010b); (10) Shapovalova et al. (2012).

and taking into account Eq. (2), we performed the least-squares fits to the linear function:

$\log E W_{\lambda}=A+\beta \log \left(F_{\text {cnt }}\right)$,

where $A=\log k$ is a normalization constant and $\beta=\alpha-1$ is the best-fitting slope of the intrinsic Beff. We calculated the Pearson correlation coefficients $r$ for each fit that indicated the rate of correlation and its statistical significance.

Furthermore, to be able to compare and discuss different objects, in Table 2 we list parameters for each galaxy such as the line FWHM, the reverberation photometric radius of the BLR $\left(R_{\mathrm{BLR}}\right)$, and the ratio of the maximum to minimum line flux for $\mathrm{H} \beta$ and $\mathrm{H} \alpha$. The last parameter is an indication of the object variability.

\section{Results and discussion}

Here we present the intrinsic Beff, shown as $\log (E W)$ versus $\log \left(F_{\mathrm{cnt}}\right)$, for the broad Balmer lines of six AGNs. Additionally, we explore how the $\mathrm{H} \alpha / \mathrm{H} \beta$ flux ratio changes as a function of the continuum in order to discuss physical background of the intrinsic Beff. We conclude this section with a comparison of the intrinsic Beff of the six monitored AGNs with the global Beff.

\subsection{Intrinsic Beff}

The available line and continuum light curves together with the corresponding intrinsic Beff, for our sample of six galaxies, are shown in Figs. 2 and 3.

In order to calculate the EW and investigate the intrinsic Beff, we used fluxes of the $\mathrm{H} \alpha$ and $\mathrm{H} \beta$ broad lines and the underlying continuum at $5100 \AA$ (and at $6200 \AA$ where it is available for the $\mathrm{H} \alpha$ line). For each object we divided the data set into two subsamples, which we denote as periods 1 and 2 . The periods are defined by the date that distinguishes between the low and high state of activity of the continuum flux at $5100 \AA$. The dividing JD and the corresponding continuum flux at $5100 \AA$ are listed 
Table 3. Parameters of the intrinsic Beff.

\begin{tabular}{|c|c|c|c|c|c|c|}
\hline Object $^{a}$ & Line $^{b}$ & $r^{c}$ & $P^{d}$ & $\beta^{e}$ & $A^{f}$ & Data sets $^{g}$ \\
\hline NGC 5548 & $\mathrm{H} \alpha$ & $\begin{array}{l}-0.997 \\
-0.984 \\
-0.927 \\
-0.959 \\
-0.913 \\
-0.936 \\
\end{array}$ & $\begin{array}{c}<0.0001 \\
<0.0001 \\
<0.0001 \\
<0.0001 \\
0.0006 \\
<0.0001\end{array}$ & $\begin{array}{l}-0.701 \\
-1.031 \\
-0.577 \\
-0.597 \\
-1.284 \\
-0.568\end{array}$ & $\begin{array}{c}-7.31 \\
-12.21 \\
-5.52 \\
-6.36 \\
-16.47 \\
-5.96\end{array}$ & $\begin{array}{c}\mathrm{p} 1 \\
\mathrm{p} 2 \\
\mathrm{p} 1+\mathrm{p} 2 \\
\mathrm{p} 1 \\
\mathrm{p} 2 \\
\mathrm{p} 1+\mathrm{p} 2\end{array}$ \\
\hline NGC 4151 & $\mathrm{H} \alpha$ & $\begin{array}{l}-0.983 \\
-0.954 \\
-0.953 \\
-0.912 \\
-0.886 \\
-0.892\end{array}$ & $\begin{array}{l}<0.0001 \\
<0.0001 \\
<0.0001 \\
<0.0001 \\
<0.0001 \\
<0.0001\end{array}$ & $\begin{array}{l}-0.922 \\
-0.691 \\
-0.580 \\
-0.617 \\
-0.513 \\
-0.392\end{array}$ & $\begin{array}{l}-9.45 \\
-6.51 \\
-4.98 \\
-6.05 \\
-4.78 \\
-3.11 \\
\end{array}$ & $\begin{array}{c}\mathrm{p} 1 \\
\mathrm{p} 2 \\
\mathrm{p} 1+\mathrm{p} 2 \\
\mathrm{p} 1 \\
\mathrm{p} 2 \\
\mathrm{p} 1+\mathrm{p} 2\end{array}$ \\
\hline 3C 390.3 & $\mathrm{H} \alpha$ & $\begin{array}{l}-0.715 \\
-0.793 \\
-0.831 \\
-0.593 \\
-0.804 \\
-0.834\end{array}$ & $\begin{array}{c}0.0006 \\
<0.0001 \\
<0.0001 \\
<0.0001 \\
<0.0001 \\
<0.0001\end{array}$ & $\begin{array}{l}-0.452 \\
-0.394 \\
-0.445 \\
-0.384 \\
-0.334 \\
-0.407\end{array}$ & $\begin{array}{c}-3.90 \\
-3.07 \\
3.81 \\
-3.52 \\
-2.82 \\
3.87\end{array}$ & $\begin{array}{c}\mathrm{p} 1 \\
\mathrm{p} 2 \\
\mathrm{p} 1+\mathrm{p} 2 \\
\mathrm{p} 1 \\
\mathrm{p} 2 \\
\mathrm{p} 1+\mathrm{p} 2\end{array}$ \\
\hline Arp 102B & $\mathrm{H} \alpha$ & $\begin{array}{l}-0.868 \\
-0.825 \\
-0.956 \\
-0.755\end{array}$ & $\begin{array}{c}<0.0001 \\
<0.0001 \\
0.0028 \\
<0.0001\end{array}$ & $\begin{array}{l}-0.912 \\
-0.858 \\
-1.310 \\
-0.774\end{array}$ & $\begin{array}{l}-11.17 \\
-11.02 \\
-18.25 \\
-9.73 \\
\end{array}$ & $\begin{array}{c}\mathrm{p} 1+\mathrm{p} 2 \\
\mathrm{p} 1 \\
\mathrm{p} 2 \\
\mathrm{p} 1+\mathrm{p} 2\end{array}$ \\
\hline Ark 564 & $\mathrm{H} \alpha$ & $\begin{array}{c}-0.651 \\
-0.820 \\
-0.884 \\
-0.591 \\
-0.485 \\
-0.701\end{array}$ & $\begin{array}{c}0.0002 \\
<0.0001 \\
<0.0001 \\
<0.0001 \\
0.0005 \\
<0.0001\end{array}$ & $\begin{array}{l}-0.523 \\
-0.807 \\
-0.680 \\
-0.701 \\
-0.747 \\
-0.577\end{array}$ & $\begin{array}{l}-5.02 \\
-9.28 \\
-7.37 \\
-8.939 \\
-9.521 \\
-7.420\end{array}$ & $\begin{array}{c}\mathrm{p} 1 \\
\mathrm{p} 2 \\
\mathrm{p} 1+\mathrm{p} 2 \\
\mathrm{p} 1 \\
\mathrm{p} 2 \\
\mathrm{p} 1+\mathrm{p} 2\end{array}$ \\
\hline E1821+643 & $\mathrm{H} \beta$ & $\begin{array}{r}-0.911 \\
-0.777 \\
-0.879\end{array}$ & $\begin{array}{l}<0.0001 \\
<0.0001 \\
<0.0001\end{array}$ & $\begin{array}{r}-0.807 \\
-0.630 \\
-0.658\end{array}$ & $\begin{array}{l}-9.38 \\
-6.86 \\
-7.25\end{array}$ & $\begin{array}{c}\mathrm{p} 1 \\
\mathrm{p} 2 \\
\mathrm{p} 1+\mathrm{p} 2\end{array}$ \\
\hline
\end{tabular}

Notes. Columns: ${ }^{(a)}$ Object; ${ }^{(b)}$ Emission line; ${ }^{(c)}$ Pearson correlation coefficient $r$; ${ }^{(d)}$ Null hypothesis probability $P$; ${ }^{(e)}$ Slope of the least-squares linear fit $\beta ;{ }^{(f)}$ Constant of the linear-fit $\log A ;{ }^{(g)}$ Data set denoted with $\mathrm{p} 1+\mathrm{p} 2$ represents full data range, $\mathrm{p} 1$ only period 1 , and $\mathrm{p} 2$ only period 2 .

in Table 2. For all objects we analyze both the overall data set and the separate periods, except for the $\mathrm{H} \alpha$ line of Arp 102B, for which we analyzed only the overall data. The results of the linear least-squares fit (correlation coefficients $r$ and corresponding $P$-value, slope $\beta$, and normalization constant $A$ ) between the EW and continuum flux for the broad $\mathrm{H} \beta$ and $\mathrm{H} \alpha$ lines are given in Table 3.

Looking into the results presented in Table 3 and Figs. 2 and 3 , we can single out several facts:

(i) Seyfert 1 galaxies NGC 4151 and NGC 5548: there is a strong correlation between $\log (E W)$ and the continuum at $5100 \AA$ for both objects. We note that the long-term monitoring of these AGNs show that they have a high degree of variability (with $F_{\max } / F_{\min } \sim 3.5-5$ in the line fluxes) and that the broad line profiles are also changing, showing shoulders and two peaks (Shapovalova et al. 2004, 2008; Bon et al. 2012; Li et al. 2016). Moreover, it was found that both of these AGNs had periodical changes in the continuum and line fluxes which indicated an orbital motion in the BLR, and consequently they were marked as SMBBH candidates (Bon et al. 2012, 2016; Popović 2012; Li et al. 2016). The intrinsic Beff slope $\beta$ in these two AGNs is significant for both broad lines in all three analyzed periods, and is always $\beta<-0.4$.

(ii) Double-peaked emitters 3C 390.3 and Arp 102B: they show a significant intrinsic Beff, but the correlation is weaker in 3C 390.3 than in Arp 102B. In addition, the correlations are weaker than in the two Seyfert 1 AGNs. There is a difference in the slope: 3C 390.3 shows a flatter slope $(\beta \sim-0.4)$ than Arp 102B ( $\beta \sim-0.9)$. We note that the long-term monitoring of these two galaxies reveals different spectral properties that can be explained as a different structure of the BLR. 3C 390.3 shows a higher rate of variability than Arp 102B (Table 2) and has a typical line variability that can be explained with the disk emission (see Shapovalova et al. 2010b; Jovanović et al. 2010; Popović et al. 2011) and perturbations in the disk, whereas this is not seen in Arp 102B (see Shapovalova et al. 2013; Popović et al. 2014). In the case of Arp 102B, the data dispersion in the Beff plot is much greater. It could be that the host-galaxy contribution to the continuum emission was larger than estimated, leading to a steeper slope of the intrinsic Beff (see Sect. 4.2). Finally, it is well known that 3C 390.3 contains various radio structures (hot spots and jets; see Leahy \& Perley 1995) which may also be sources of additional optical continuum emission, 

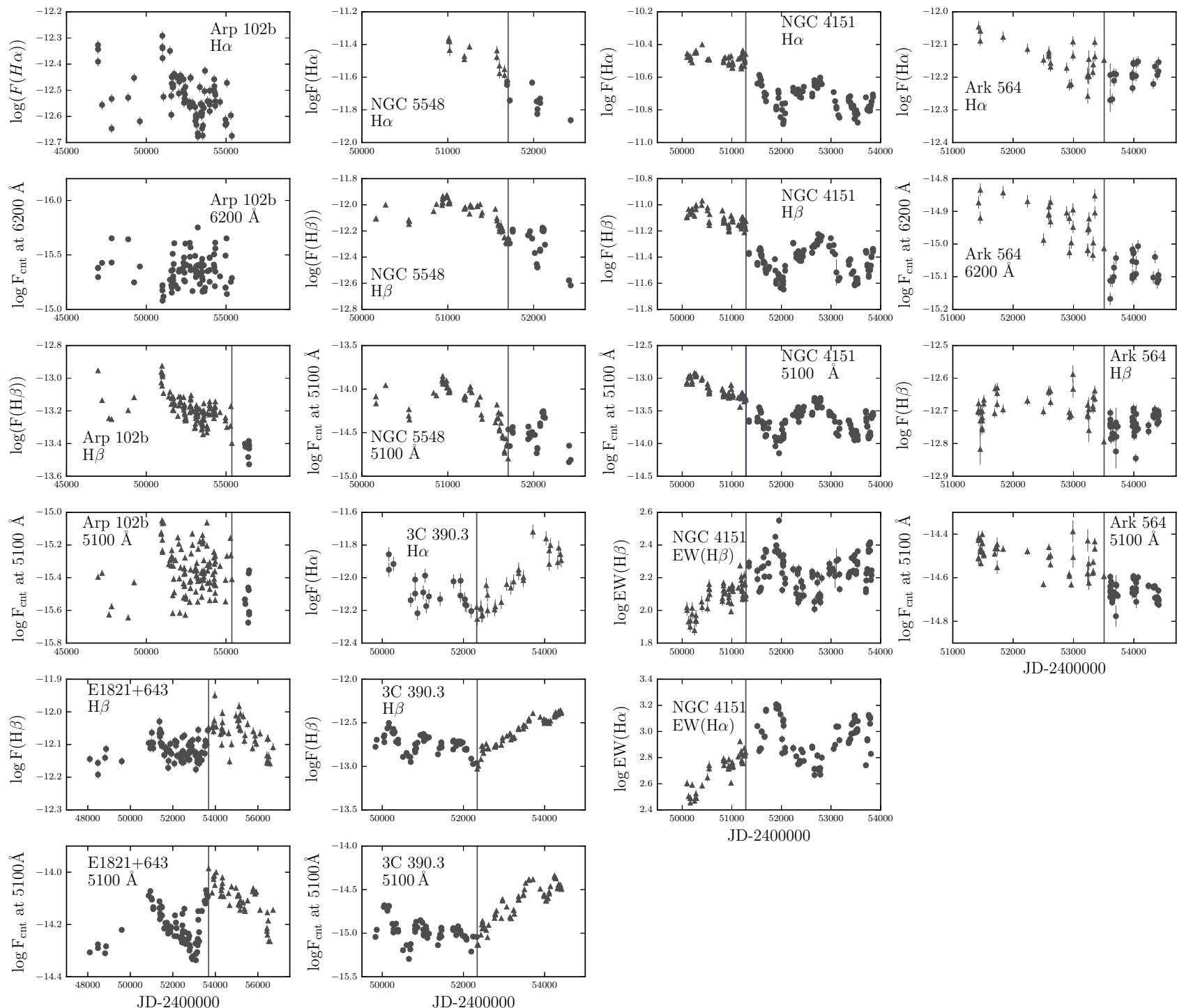

Fig. 2. Light curves of the broad $\mathrm{H} \alpha$ and $\mathrm{H} \beta$ emission line fluxes, together with the continuum fluxes at $5100 \AA$ and $6200 \AA$ in logarithmic scale for the sample of six AGNs (object name and line/continuum denoted on each plot). Data are divided on the basis of the high-flux (triangles) and low-flux (full circles) states. Error bars are also shown, unless smaller than the marker.

since Arshakian et al. (2010) showed that the radio emission in 3 C 390.3 is correlated with the optical continuum.

(iii) NLSy 1 Ark 564: the amplitude of variability of Ark 564 is small, and consequently the corresponding dynamical range (EW versus L5100) is relatively small, which may flatten the Beff slope. However the intrinsic Beff in this AGN is clearly detected. We note that the variability in high-energy light curves shows remarkably high amplitude (see, e.g., Kara et al. 2013). Some NLSy 1 are peculiar AGNs, the variability in the optical spectra shows many flare-like features (see, e.g., Shapovalova et al. 2012), and they may be $\gamma$-ray emitters, indicating the presence of relativistic jets or outflows like those in blazars (see Yang \& Zhou 2015). Ark 564 shows a weak correlation between $\log (E W)$ and continuum (Table 3 ) and there is a difference in the slope and correlation coefficient for $\mathrm{H} \alpha$ and $\mathrm{H} \beta$, which is expected since our longterm monitoring showed a weak correlation between $\mathrm{H} \alpha$ and $\mathrm{H} \beta$ fluxes (see Shapovalova et al. 2012).

(iv) High-luminosity quasar E1821+643: it is clear that E1821+643 exhibits significant intrinsic Beff as seen in other objects in our sample. The quasar E1821+643 also shows change in the slope of the intrinsics Beff.

\section{2. $H \alpha / H \beta$ flux ratio versus continuum}

In addition to the intrinsic Beff, we explore the $\mathrm{H} \alpha / \mathrm{H} \beta$ flux ratio as a function of the continuum flux. Assuming a pure photoionization model, we can expect that the $\mathrm{H} \alpha / \mathrm{H} \beta$ broad line ratio has an average value of $\sim 3$ (Osterbrock \& Ferland 2006), as observed in a number of AGNs (Dong et al. 2008). On the other hand, the ratio of $\mathrm{H} \alpha / \mathrm{H} \beta$ can give an indication of some physical processes in the BLR (see Popović 2003; Popović et al. 2008; Ilić et al. 2012) that might be connected with the intrinsic Beff.

The $\mathrm{H} \alpha / \mathrm{H} \beta$ line flux ratios versus the continuum flux at $5100 \AA$ for all objects, except E1821+643 for which the $\mathrm{H} \alpha$ line is not available, are shown in Fig. 4. We also plot in Fig. 4 (bottom right panel) the $\mathrm{H} \alpha / \mathrm{H} \beta$ versus continuum luminosity at $5100 \AA$ for a subsample of 4800 objects (for which $\mathrm{H} \alpha, \mathrm{H} \beta$, and continuum at $5100 \AA$ luminosities were available) from the 

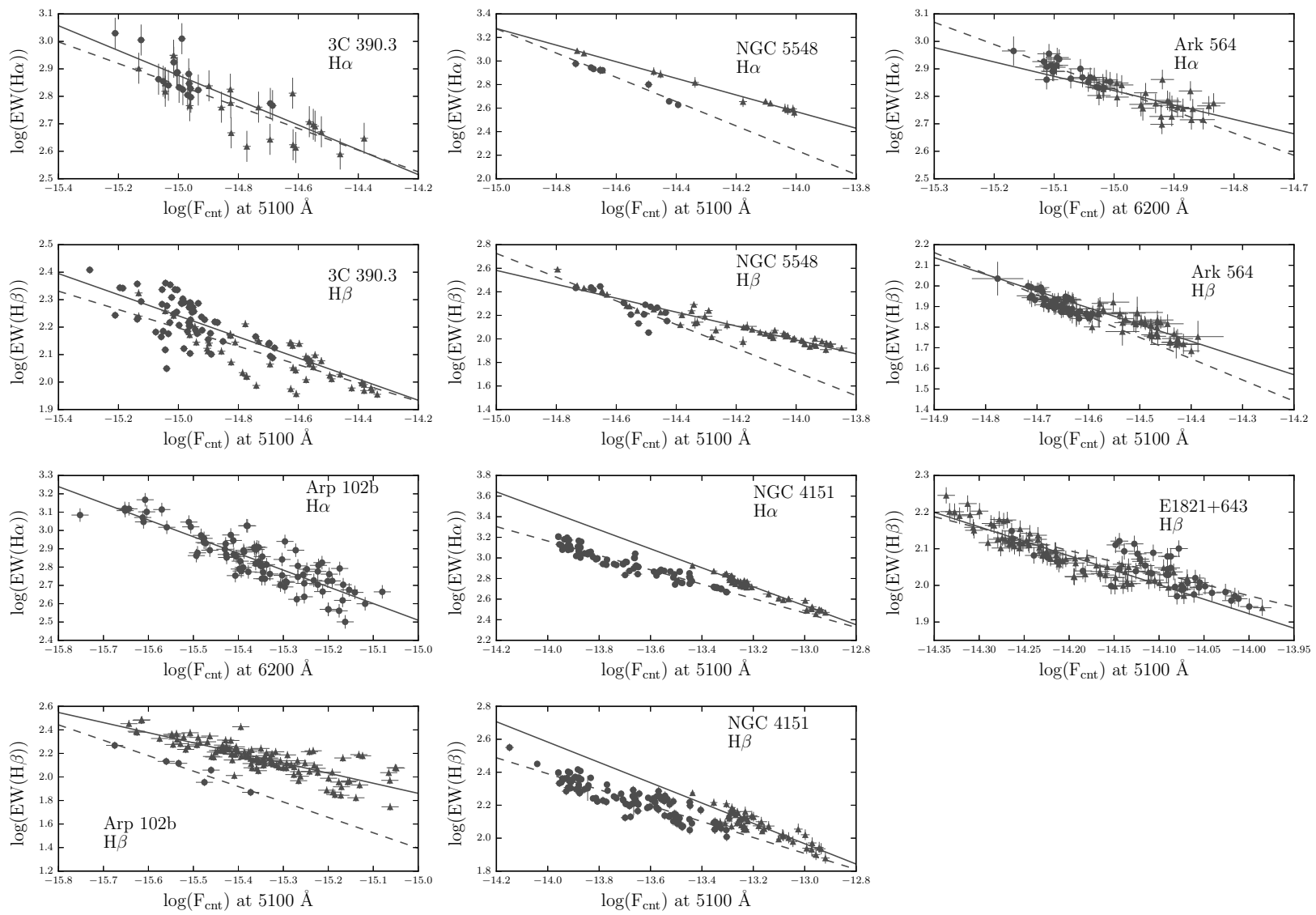

Fig. 3. Intrinsic Beff for the sample of six AGNs (name and line denoted on each plot). Data are divided and marked in the same way as in Fig. 2. Solid and dashed lines represent the best fits to the data.
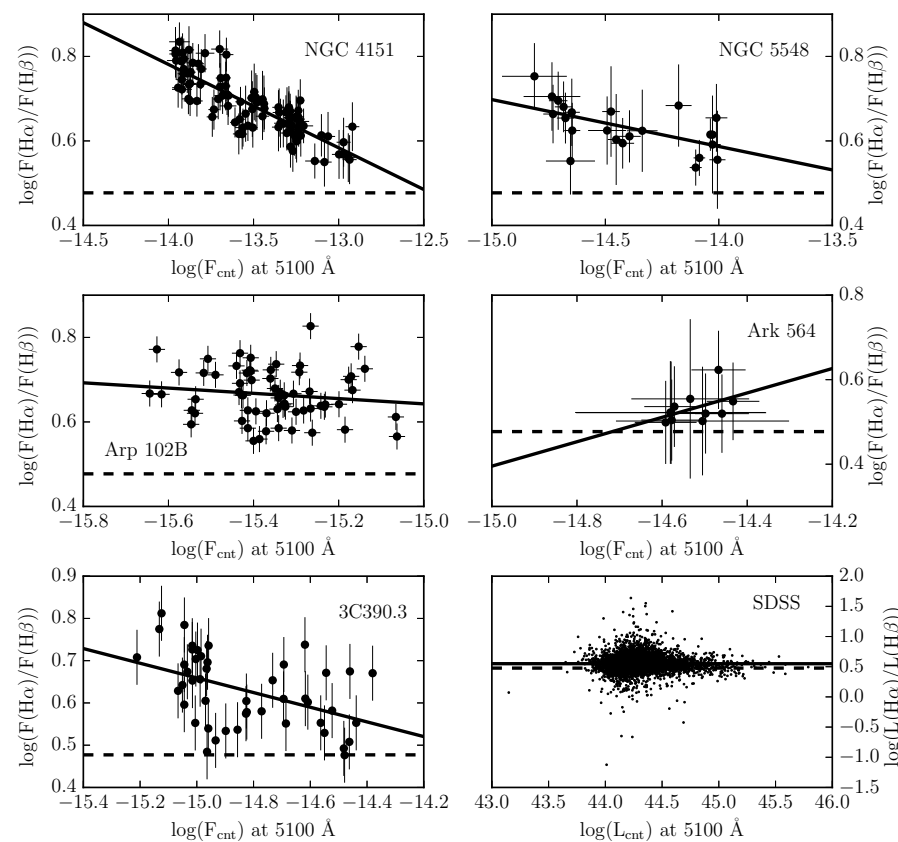

Fig. 4. $\mathrm{H} \alpha / \mathrm{H} \beta$ flux ratio vs. the continuum flux at $5100 \AA$ in logarithm scale, for the five AGNs in our sample for which both $\mathrm{H} \alpha$ and $\mathrm{H} \beta$ fluxes are available (name denoted on each plot), and for a sample of 4800 SDSS AGNs from Shen et al. (2011; bottom right panel). The solid line represents the best fit of the observed data and the dashed line represents the typical ratio $\mathrm{H} \alpha / \mathrm{H} \beta \approx 3$ expected in the case of pure photoionization.
SDSS database studied by Shen et al. (2011). It can be seen in Fig. 4 that the behavior of the $\log (\mathrm{H} \alpha / \mathrm{H} \beta)$ versus continuum is different for different AGNs in our sample, but it is always above 3 (dashed line in all panels). It seems that the $\mathrm{H} \alpha / \mathrm{H} \beta$ anti-correlates with the continuum emission (solid line in the top left panel) only in NGC 4151. For the rest of the AGNs there is no significant (anti-)correlation with the continuum, and the $\mathrm{H} \alpha / \mathrm{H} \beta$ ratio has different responses to the continuum: i) in the case of NGC 5548 there is a similar trend to that in the case of NGC 4151, but with higher scatter; ii) in Arp 102B and Ark 564 the ratio is more or less constant; and iii) in 3C 390.3 the change is irregular, which may be due to two different variability phases of the broad line profiles, one caused by the central source outburst and second caused by the change in the disk structure (see Jovanović et al. 2010; Popović et al. 2011). Finally, the averaged $\mathrm{H} \alpha / \mathrm{H} \beta$ ratio (solid line in the bottom right panel) in the sample of 4800 AGNs is almost 3.

The most important aspect to be noted is that although the intrinsic Beff is present in all objects, the $\mathrm{H} \alpha / \mathrm{H} \beta$ flux ratio varies differently with the continuum for different AGNs in our sample. In the case of NGC 4151 and NGC 5548, this ratio tends to 3 for the highest flux states, and for the case of Ark 564 is almost constant and close to 3 , which is expected as the photoionization is dominant. However, in the case of Arp 102B and 3C 390.3 (AGNs with double-peaked lines) this ratio as a function of the optical continuum seems to be more complex.

Finally, we note that the $\mathrm{H} \alpha / \mathrm{H} \beta$ line flux ratio in AGNs may be complicated and in general is not fully understood; it may be also affected by the reddening and optical depth effect. However, 


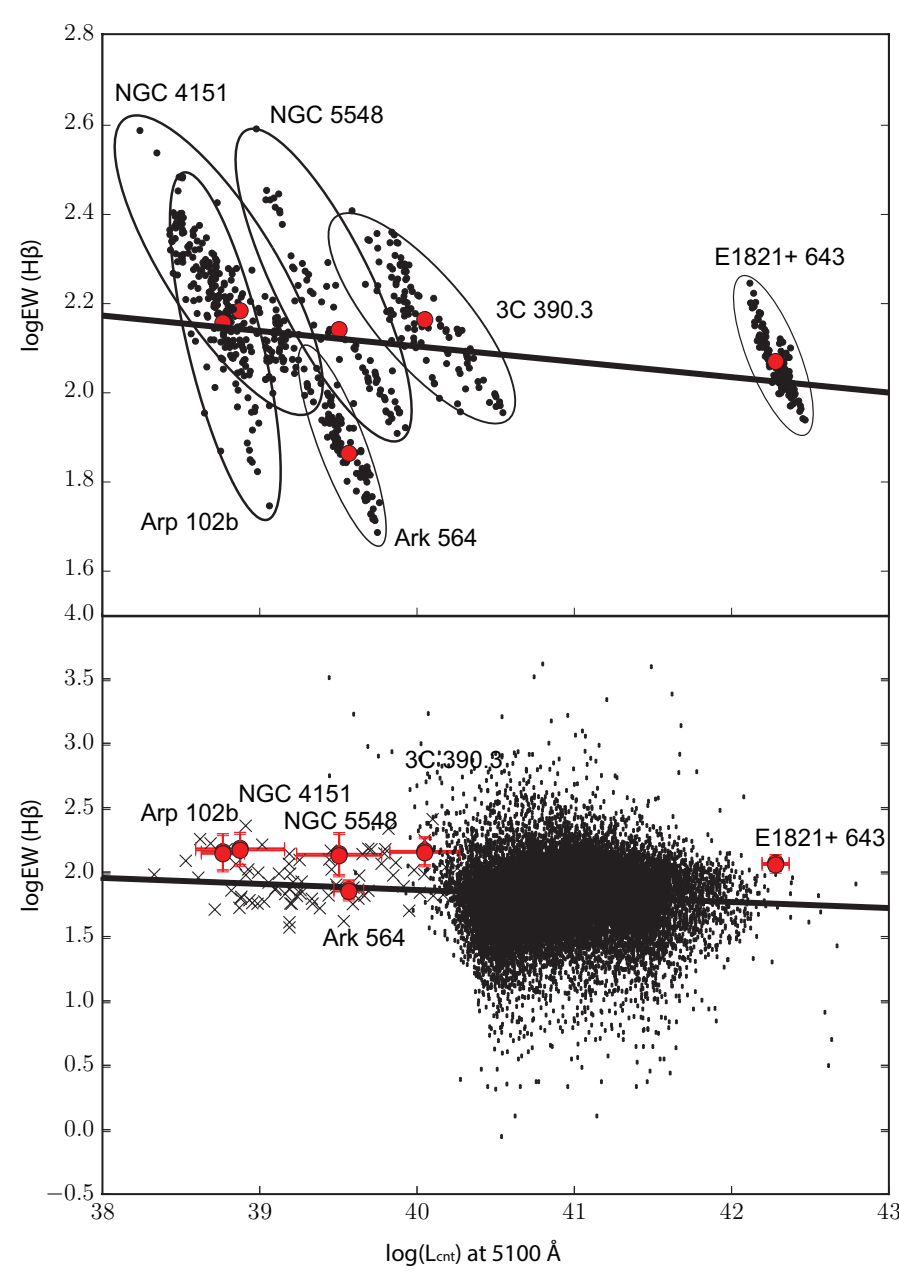

Fig. 5. Global Beff for $\mathrm{H} \beta$. Top: for our samples of six AGNs, we plot the $\mathrm{H} \beta$ line EW and continuum luminosity at $5100 \AA$ (dots surrounded with contours). The corresponding averaged line EW and continuum luminosity is denoted with a circle for each object. The best-fitting of all the measurements for the six objects is shown with the solid line. Bottom: averaged values of the six studied objects (circles), together with the sample of 21416 AGNs measured by Shen et al. (2011; dots) and 90 low-luminosity AGNs from La Mura et al. (2007; crosses). The best-fitting of all data is shown with the solid line.

in the global picture (see bottom right panel in Fig. 4), no trend similar to the trend seen in NGC 4151 is present between the $\mathrm{H} \alpha / \mathrm{H} \beta$ ratio and the luminosity.

\subsection{Global Beff}

It is interesting to consider the intrinsic Beff in the context of the global one, since the broad Balmer lines do not show global Beff (see, e.g., Kovačević et al. 2010; Popović \& Kovačević 2011).

To compare the intrinsic and global Beff, we plot the EW of the $\mathrm{H} \beta$ line versus the continuum luminosity at $5100 \AA$ for all measurements of the six considered AGNs (dots surrounded by contours in the top panel of Fig. 5). The circles in both panels in Fig. 5 represent the average value of EW and continuum. From this plot, it seems that the global Beff can hardly hold in the broad component of the $\mathrm{H} \beta$ emission line when all six objects are considered (full line in Fig. 5).

This situation is even more noticeable when these data are compared with the results obtained for a large sample of 21416 sources from Shen et al. (2011), and with 90 low-luminosity sources from La Mura et al. (2007; Fig. 5, bottom panel). As Fig. 5 clearly illustrates, even though the EWs measured in this large sample are fully consistent with those estimated from the variability monitoring campaign, no evidence of a significant global Beff in the broad component of $\mathrm{H} \beta$ is detectable (slope $\beta=-0.0467$ ).

We can conclude that according to the results for the six monitored AGNs and for the much larger sample of single-epoch observations (Fig. 5), we could hardly detect significant global Beff in the broad component of $\mathrm{H} \beta$. This agrees with previous findings, which concluded that no global Beff was present in the broad Balmer lines (Dietrich et al. 2002; Kovačević et al. 2010).

\section{Physical interpretation of the intrinsic Beff}

Before discussing the possible physical explanations of the intrinsic Beff, we summarize several facts noted above:

(i) There is a significant intrinsic Beff in all six AGNs, which makes only little difference between the different types of AGNs. The broad line profiles of the considered AGNs are quite different, and the structure of the BLRs seems to be different. Consequently, the geometry of the BLR probably does not have a significant impact on the presence of the intrinsic Beff;

(ii) In an individual AGN, there are different Beff slopes in different periods defined on the basis of the continuum flux (going from the high to the low activity phase, or vice versa). This may be connected with the nature of the ionizing continuum source and consequently with the physics of the BLR;

(iii) There is a different response of the $\mathrm{H} \alpha / \mathrm{H} \beta$ ratio for different objects. This may be caused by the different mechanism of the optical continuum emission, but also by the change in the structure of the line-emitting disk-like region (e.g., in 3C 390.3; see Popović et al. 2011) or spatially extended BLR (see Goad \& Korista 2014);

(iv) The intrinsic Beff is not connected with the global Beff, i.e., even if the global Beff is not present in the broad Balmer lines, the intrinsic Beff is significant.

Moreover, Seyfert 1 and NLSy1 AGNs show different spectral characteristics caused by different inclinations, accretion rates, and other physical properties (see, e.g., Sulentic et al. 2000, 2009; Zhou et al. 2006; Shen \& Ho 2014; Bisogni et al. 2017, etc.), which should probably result in a different intrinsic Beff. But this is not the case when comparing the intrinsic Beff of Ark 564 (NLSy1) and other Type 1 AGNs in our sample. Therefore, we can conclude that the intrinsic Beff is probably not connected with the orientation or the accretion rate. However, we should note that we have only one NLSy1 in our sample, and the influence of the accretion rate should be checked on a larger sample of NLSy1 AGNs.

We considered AGNs with quite different broad line characteristics. First, in two Seyfert 1 galaxies NGC 4151 and NGC 5548 in addition to the line and continuum variability, the shape of line profiles has also varied significantly, especially in the case of NGC 4151 (Shapovalova et al. 2010b). This variability could be associated with the contribution of outflows (Ilic et al. 2010) or even with the SMBBH (Bon et al. 2012; Li et al. 2016). Then, 3C 390.3 seems to be representative of the disk-like BLR (Popović et al. 2011), while this is not clear in the case of Arp 102B (Popović et al. 2014), even though they both have double-peaked broad Balmer lines. Additionally, we have 


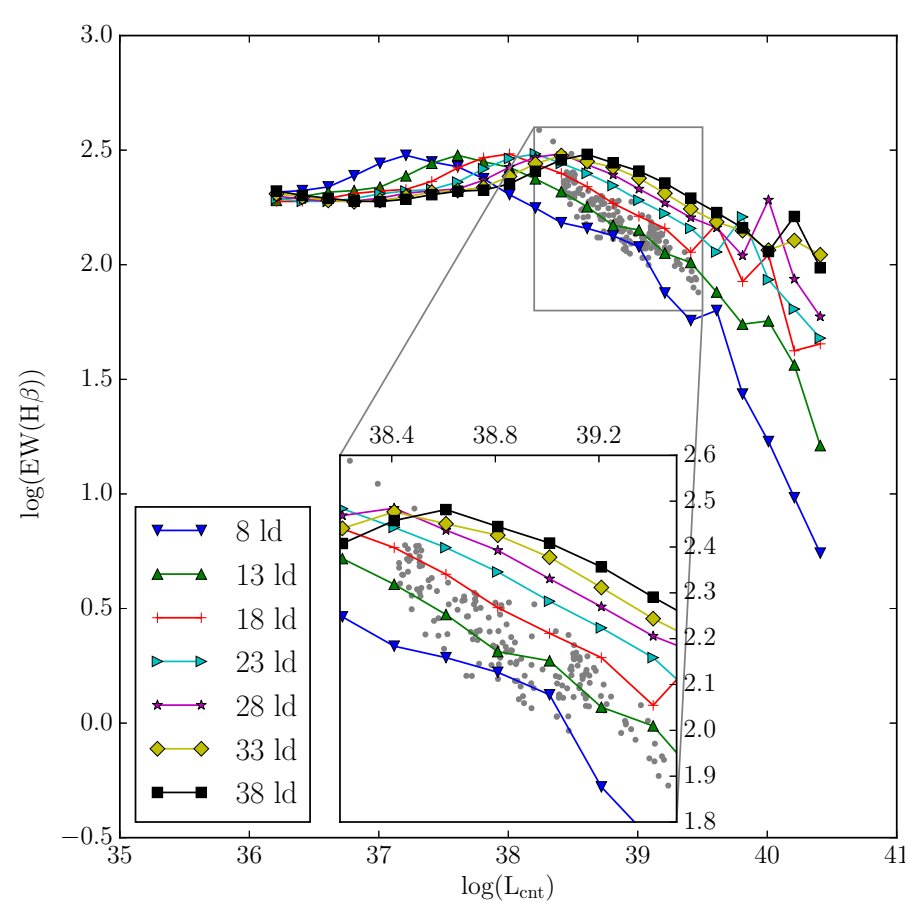

Fig. 6. Correlation of the $\mathrm{EW} \mathrm{H} \beta$ with the incident luminosity of the continuum at $5100 \AA$ for different $R_{\mathrm{BLR}}$ (denoted in the bottom left), obtained from the Cloudy simulations. Circles represent observed intrinsic Beff for the $\mathrm{H} \beta$ line of NGC 4151 with continuum fluxes corrected for the host-galaxy contribution (zoomed-in for better visibility).

the extremely luminous quasar E1821+643 with strongly redshifted broad lines, which is regarded as a SMBBH candidate (see Shapovalova et al. 2016, and references therein). Finally, Ark 564 is an NLSy1 galaxy whose BLR might have a different geometry. Hence, a question arises: What do all of these AGNs that can produce the intrinsic Beff have in common?

To find an answer to this question, we should explore the physical causes connected with the central continuum source and physical properties in the BLR. We consider the photoionization, recombination, and collision effects, taking into account that for higher ionization parameters, the photoionization followed by recombination is more important in the BLR. However at higher temperatures or in the case of low-ionization parameters we can expect that the collisional excitation becomes important (Osterbrock \& Ferland 2006; Popović et al. 2008; Ilić et al. 2012). Apart from these two effects, the radiative-transfer effects in Balmer lines can affect the broad Balmer line ratio. However, we can assume that photoionization is the dominant process in the BLR, and so to explain the observed intrinsic Beff we start with photoionization.

\subsection{Pure photoionization model}

To explore the physical background of the intrinsic Beff, we first generated several grids of the BLR models using the photoionization code Cloudy (version 13.03, described in Ferland et al. 2013).

We modeled a simple single BLR cloud in an open geometry, as plane parallel slabs shed by the continuum of an AGN, defined through the bolometric luminosity $\left(L_{\mathrm{bol}}\right)$, the radius of the BLR $\left(R_{\mathrm{BLR}}\right)$, and the hydrogen density $\left(n_{\mathrm{H}}\right)$. We used the column density value of $10^{23} \mathrm{~cm}^{-2}$ for the stopping criteria.
The hydrogen density was fixed to $n_{\mathrm{H}}=10^{11} \mathrm{~cm}^{-3}$ and the shape of the ionizing continuum was chosen as the table of the characteristic continuum for an AGN (Ferland et al. 1998). To produce one grid of models we varied $\log L_{\text {bol }}$ within the range [41-45.2] $\mathrm{erg} \mathrm{s}^{-1}$ with an intermediate step of 0.2 dex.

We attempted to explain the intrinsic Beff in NGC 4151 that had a relatively compact BLR (see Peterson \& Cota 1988; Clavel et al. 1990; Maoz et al. 1991; Bentz et al. 2006a; Shapovalova et al. 2008); therefore, we produced several grids of models by varying the $R_{\mathrm{BLR}}$ in the range of 8-38 light days (1.d.), with a step of 5 l.d. The EW was calculated in a similar way using Eq. (2), but in this case we used luminosities instead of fluxes. In Fig. 6 we plot the $\operatorname{EW}(\mathrm{H} \beta)$ versus the incident continuum luminosity at $5100 \AA$ from the output of the Cloudy code calculation. We explore their correlation in order to assess whether an intrinsic Beff is predicted by a simple photoionization theory, and to identify the origin of different Beff slopes of a single source. We note that detailed studies of variability of broad lines under photoionization processes are given in Korista \& Goad (2004) and Goad \& Korista (2014), where different effects are considered. Here, we used a simple model in order to reproduce the effects observed in the case of NGC 4151.

\subsubsection{NGC 4151: a study case}

We tried to reproduce the observed Beff of NGC 4151, as it is shown in Fig. 3 panel 7, through our set of photoionization models (see Fig. 6). Figure 6 shows that a strong intrinsic Beff (with a slope of -0.331 and -0.205 ) is present for clouds located close to the source, i.e., for models obtained with $R_{\mathrm{BLR}}=8 \mathrm{l}$.d. and $R_{\mathrm{BLR}}=13$ l.d. respectively. At larger distances (i.e., larger BLR radius), for the low-luminosity states, the EW of $\mathrm{H} \beta$ is nearly constant, but for higher luminosity states there is a significant drop in EW and an intrinsic Beff occurs. Taking into account that the modeled clouds have fixed column density (which determines the cloud size), the emitted line intensity (which responds to an increasing continuum flux) will grow until there is no neutral gas in the cloud that can be further ionized. This corresponds to the nearly constant EW that is observed in the low-luminosity state (Fig. 6). When the flux is high enough to completely ionize the cloud, the line intensity can no longer grow with the continuum and a sharp drop in the EW is present. Since the flux grows linearly with luminosity, but decreases with the square of the cloud distance, the drop will appear at higher luminosities in more distant clouds. On the other hand, the cloud will see a large flux at low luminosity when it is closer to the source (so EW will be larger at small radii), but it will also saturate sooner; i.e., when the luminosity increases, the EW of a closer cloud decreases before the EW of a farther one. Figure 6 implies that one of the reasons for the variability of the intrinsic Beff in NGC 4151 (illustrated in Fig. 3 panel 8) could be a change of the line emission site in the BLR. A comparison of the models with observed continuum luminosities (plotted as circles in Fig. 6), suggests that agreement can only be achieved over a limited range of BLR radii.

Based on the simulations, the possible observed variability in the intrinsic Beff could be explained with the changes in the size of the emitting region and with different gas layers contributing to the various components of the line profile, depending on the strength of the incident continuum. However, the BLR of NGC 4151 seems to be very complex (Ilić et al. 2008; Shapovalova et al. 2008, 2010b; Bon et al. 2012) and we have to consider that photoionization might not be the only 
mechanism of ionization (Shapovalova et al. 2008). One problem of particular interest in this AGN is the determination of its BLR size. A number of reverberation studies have shown inconsistent results for the BLR dimension, finding that the BLR may extend from less than 2 1.d. (Shapovalova et al. 2008) to 4-10 1.d. (Peterson \& Cota 1988; Clavel et al. 1990; Maoz et al. 1991; Bentz et al. 2006a), but all of these studies agreed that the BLR was very compact. Our models show that the intrinsic Beff strength could be explained with photoionization, but only if the BLR size is between 8 and 13 l.d. (see Fig. 6), in agreement with the previous results of the compact BLR. We note here that Shapovalova et al. (2008) showed, using the grid of Cloudy models given by Korista et al. (1997), that the observed $\mathrm{H} \beta$ line fluxes could be explained only by a larger BLR ( 30 1.d., which can emit an intensive line flux that is far larger than the reverberation estimates) or by an additional ionizing continuum responsible for the line production, which comes from either outflows or jets.

Although the observed slope of the intrinsic Beff for the $\mathrm{H} \beta$ line in NGC $4151(\mathrm{p} 1+\mathrm{p} 2:-0.392)$ is close to the values predicted by models with $R_{\mathrm{BLR}}=8$ and 13 l.d. $(-0.331$ and -0.205 , respectively; see Fig. 6), the models do not fit the observations exactly, but these simple models give us an idea of the possible reasons for changes in the intrinsic Beff slope. The idea is that the emission line site in the BLR moves on radii that are farther away with an increase in the continuum luminosity; i.e., a "breathing effect" exists in the BLR of NGC 4151. A similar effect was detected in the BLR of NGC 5548 (Cackett \& Horne 2006).

\subsubsection{Photoionization and the intrinsic Beff: possible scenario and limits}

The proposed simple photoionization models provide a possible scenario which could explain the intrinsic Beff: as the ionization continuum increases, the broad line emission becomes saturated and the inner layers of the BLR become optically thin for the continuum radiation. As a consequence, the site of line formation moves to more distant gas layers, which react to the changes in the incident photon flux with a different response.

However, the simple photoionization models have several limitations that we discuss here. The first is the ratio of Ly $\alpha$ to $\mathrm{H} \beta$ obtained from our simulations that is above 30 , in contradiction with the measured values around 20-25 (Penston et al. 1979; Netzer et al. 1995). A second point is that we cannot reproduce the $\mathrm{H} \alpha / \mathrm{H} \beta$ ratio. As can be seen in Fig. 7, the modeled ratios do not fit the observed $\mathrm{H} \alpha / \mathrm{H} \beta$ ratios (circles), especially in the low-activity state of NGC 4151. In contrast to the usual nebular case, the $\mathrm{H} \alpha / \mathrm{H} \beta$ ratio can span a wide range of values in a BLR environment; for example, several Type 1 AGNs exhibit ratios $\mathrm{H} \alpha / \mathrm{H} \beta>3$. In BLR clouds there are several factors that affect this ratio: superthermal electrons due to the high-energy AGN radiation field, trapped Ly $\alpha$ photons that overpopulate hydrogen in its level 2, enhanced recombination due to high density, and a possible contribution from an intrinsic extinction. All these effects combine in such a way that we expect higher $\mathrm{H} \alpha / \mathrm{H} \beta$ ratios with respect to a Case $\mathrm{B}$ situation whenever the cloud has a significant fraction of neutral gas. Most of the models actually appear to predict a very high $\mathrm{H} \alpha / \mathrm{H} \beta$ ratio in the low-flux regime, perhaps indicating that the high-order Balmer lines are destroyed in neutral gas, favoring $\mathrm{H} \alpha$ much like what happens in the Lyman series at lower densities. When the flux grows (because of the luminosity increase, but the effect naturally occurs at higher luminosities for more distant clouds), the destruction

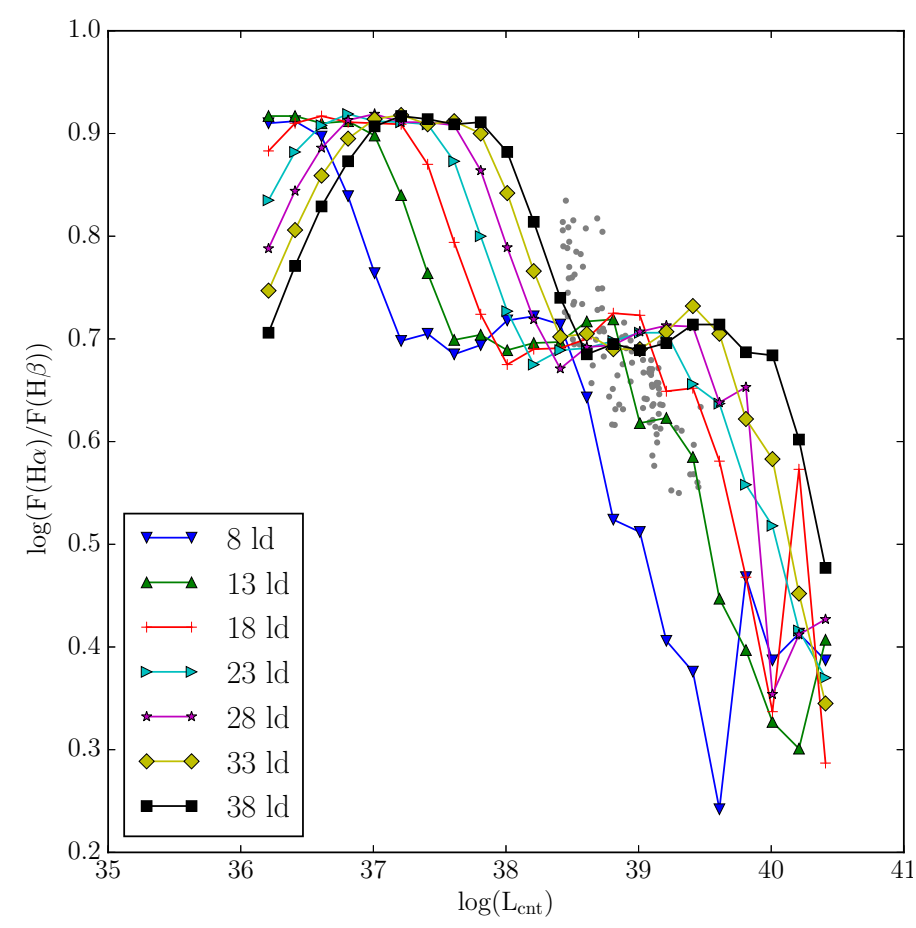

Fig. 7. $\mathrm{H} \alpha / \mathrm{H} \beta$ ratio vs. the continuum at $5100 \AA$ for different $R_{\mathrm{BLR}}$ sizes (denoted in the bottom left) compared with the observed ratio for NGC 4151 (circles).
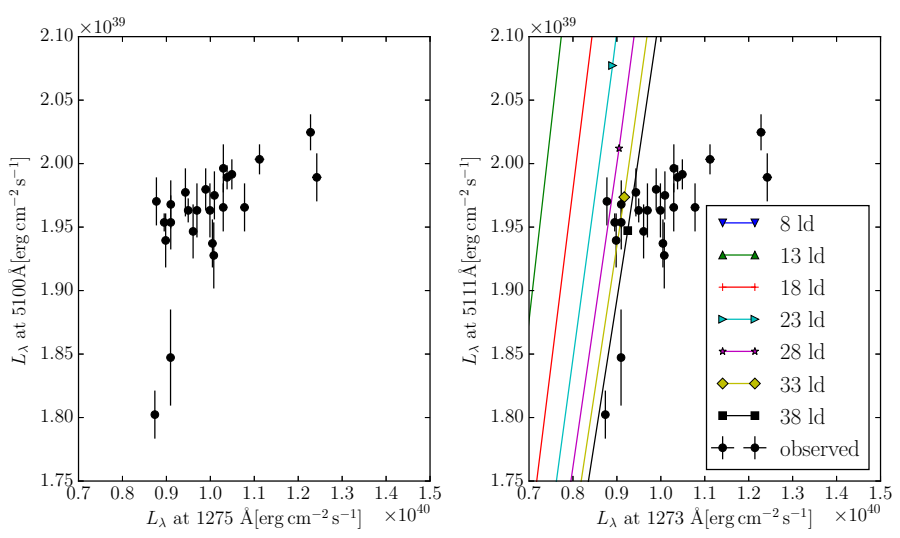

Fig. 8. Left: observed continuum flux at $1275 \AA$ vs. the flux at $5100 \AA$ for NGC 4151 obtained during short-time period (see text for details). Right: modeled continuum flux at $1275 \AA$ vs. the flux at $5100 \AA$ (solid lines for different $R_{\mathrm{BLR}}$ sizes, denoted in the bottom left) compared to the observed values (full circles).

of high-order Balmer photons becomes ineffective and the ratio reaches a quasi-constant value (as it can be seen in Fig. 7). In our models, this occurs approximately in the same luminosity range where the EW achieves its maximum, meaning that this is the range of optimal conditions for the emission of $\mathrm{H} \beta$. If, however, the continuum grows at even higher fluxes, the $\mathrm{H} \alpha / \mathrm{H} \beta$ ratio experiences a quick drop. This effect can occur because clouds in such conditions rapidly become inefficient line emitters, leading to emission line ratios that tend to even out.

A further concern for simple photoionization calculations is the nontrivial relationship existing between the optical continuum and the intensity of the ionizing radiation field. We tested the relation between the ionizing flux in the UV and optical spectral bands in the case of NGC 4151, using the data from short-timescale multiwavelength observations carried out 
by Crenshaw et al. (1996; UV) and Kaspi et al. (1996; optical) in December 1993. In Fig. 8, we show the relation between the continua at $1275 \AA$ and $5100 \AA$. We calculated the Pearson correlation coefficient $r=0.577$ with $P=0.003$ and we performed the best linear fit obtaining a slope of 0.028 . Additionally, we compared the UV versus optical continuum with those from photoionization models. As can be seen in Fig. 8, although there is some degree of correlation between the two continua, strong variations in the UV are only followed by weak changes in the optical domain. Furthermore, in the low-luminosity stage there appears to be a large break, which would be unexpected in the case of pure photoionization, but could be explained by an optical continuum component that does not relate with UV. This becomes apparent when the AGN is in its low-activity stage and the ionizing continuum is not dominating the overall spectrum.

It seems that the photoionization model, which changes the BLR size, can explain the intrinsic Beff, but there are several observational facts (the $\mathrm{H} \alpha / \mathrm{H} \beta$ ratio and UV versus optical continuum variability) that cannot be explained by this model with the same parameters. These differences might be accounted for by the presence of effects that were not considered in the photoionization calculations.

\subsection{Additional continuum emission}

First, we recall some results obtained from variability investigations of the AGNs in our sample. We start with the conclusions in Shapovalova et al. (2008), where the photoionization model could not explain the correlation between the line and continuum flux in NGC 4151 since a saturation in the broad line flux was observed in the high continuum state (see Fig. 10 in Shapovalova et al. 2008, and discussion in the paper). It seems that an extra ionizing continuum source is present in NGC 4151. We can also see in Fig. 8 that there is no significant response of the optical to the UV continuum during the short period. In addition, in the case of 3C 390.3 it was discovered that the variability in the optical continuum had a strong correlation with that in the radio continuum (see Arshakian et al. 2010). More recently Goad \& Korista (2015) considered the EW variation of NGC 5548, and in order to explain the EW variability they invoked a smaller value of the incident ionizing photon flux that was different from the observed UV continuum flux and typical models of the continuum SED.

Taking into account all the above, we should consider a possibility that the intrinsic Beff may be caused by a portion of additional continuum emission that is not connected with the ionization continuum. The total optical continuum can be considered as the sum of the optical continuum that follows the SED of the UV ionizing continuum, and a fraction of the additional optical (nonionizing) continuum that can be produced by different processes. The luminosity of broad lines $L_{\text {line }}$ is a function of the ionizing continuum (see, e.g., Korista \& Goad 2004) as

$L_{\text {line }}=A \cdot L_{\text {ion }}^{\alpha}$,

where $\alpha$ represents an emission-line responsivity, giving

$\log E W \sim(\alpha-1) \log L_{\text {ion }}$.

The expected intrinsic Beff slope $\beta$ should not be too high; i.e., assuming photoionization it follows that $R_{\mathrm{BLR}} \sim L^{0.5}$ (Bentz et al. 2006b), and using a rough estimate that $L_{\text {line }} \sim R_{\mathrm{BLR}}^{3}$ (Osterbrock \& Ferland 2006), we obtain $\alpha \sim 1.5$, giving $\beta \sim 0.5$. In contrast, we infer that in some cases $\beta$ is around -1 (see Table 3), indicating very low values of $\alpha$. This may imply that the reprocessing of the ionizing continuum by the line emission is very low and part of this continuum is transformed into the optical one, which is observed as the additional continuum in the optical band. This is also in agreement with the results obtained for NGC 5548 broad line variability by Goad \& Korista (2015).

A part of the additional optical continuum may be emitted partially from the BLR itself in free-bound and free-free transitions since, with the increase in the ionization continuum, we can expect an increase in the rate of ionization, i.e., the number density of electrons and ions. However, the additional continuum could also come from jet or outflow, as in the case of 3C 390.3 (see Arshakian et al. 2010). We note here that the "additional continuum" problem has to be considered in more detail, taking into account the X-ray, UV, and optical spectra of several AGNs, which is beyond the scope of this paper.

\section{Conclusions}

The intrinsic Baldwin effect in the broad $\mathrm{H} \beta$ and $\mathrm{H} \alpha$ lines of a sample of six Type 1 AGNs was studied. In our sample, we considered two Seyfert 1 galaxies (NGC 5548, NGC 4151), two AGNs with very broad double-peaked lines (3C 390.3 and Arp 102B), one NLSy 1 galaxy (Ark 564), and one highluminosity quasar (E1821+643). We used data obtained from a single long-term monitoring campaign (Shapovalova et al. 2004, 2008, 2010a, 2012, 2013, 2016), which made our sample uniform and consistent. We compared observed data with the results obtained with the numerical code Cloudy, and also discussed the global Beff of these six AGNs, combined with the sample of 21500 objects from the SDSS database. We can outline the following conclusions:

1. The intrinsic Beff is present in broad Balmer lines of six Type 1 AGNs studied in this paper. The shift and the change in the slope of the intrinsic Beff is observed in all the considered AGNs. Taking into account that the AGNs in the studied sample have different line shapes that indicate different BLR geometries, the intrinsic Beff is probably not caused by the geometry of the BLR. However, this should be tested on larger samples of different AGNs.

2. The lack of a significant global Beff for the broad Balmer lines is confirmed, and there is no connection between the global and intrinsic Beff.

3. Using the Cloudy code to build a simple model of line emitting clouds in the BLR of NGC 4151, we found that the photoionization models were able to reproduce the intrinsic Beff, which also suggests that the variation in the Beff slope in NGC 4151 might be due to a change of the site of line formation in the BLR.

However, pure photoionization cannot explain the $\mathrm{H} \alpha / \mathrm{H} \beta$ ratio and the $\mathrm{Ly} \alpha / \mathrm{H} \beta$ ratio in this object. Consequently, the additional optical (nonionizing) continuum that may be emitted from the BLR or from outflows can be an alternative, but the natures of these two possibilities have to be investigated in greater detail.

Although our considerations of additional continuum components affecting the Beff are mainly based on indirect evidence, we recall that deviations from a linear line response to the underlying continuum have been observed in NGC 4151 in both its highest and lowest activity. In order to constrain the contribution of nonionizing continuum components, it is important to study AGNs in their maximum and their minimum activity when deviations of broad line responses are expected to be more evident. 
Acknowledgements. We are grateful to the anonymous referee for carefully reading our paper and giving comments and suggestions that greatly improved our paper. This work is a part of the project (176001) "Astrophysical Spectroscopy of Extragalactic Objects", supported by the Ministry of Education, Science and Technological Development of Serbia and the project "Investigation of supermassive binary black holes in the optical and X-ray spectra" supported by the Ministry of Science and Technology of R. Srpska. Also, this work was supported by INTAS (grant N96-0328) and RFBR (grants N97-02-17625 N00 02-16272, N03-02-17123, 06-02-16843, N09-02-01136,12-02-00857a, 12-0201237a, N15-02-02101). The work was partly supported by the Erasmus Mundus Master Program, AstroMundus.

\section{References}

Arshakian, T. G., León-Tavares, J., Lobanov, A. P., et al. 2010, MNRAS, 401, 1231

Bachev, R., Marziani, P., Sulentic, J. W., et al. 2004, ApJ, 617, 171

Baldwin, J. A. 1977, ApJ, 214, 679

Baskin, A., \& Laor, A. 2004, MNRAS, 350, L31

Bentz, M. C., Denney, K. D., Cackett, E. M., et al. 2006a, ApJ, 651, 775

Bentz, M. C., Peterson, B. M., Pogge, R. W., Vestergaard, M., \& Onken, C. A 2006b, ApJ, 644, 133

Bian, W.-H., Fang, L.-L., Huang, K.-L., \& Wang, J.-M. 2012, MNRAS, 427, 2881

Bisogni, S., Marconi, A., \& Risaliti, G. 2017, MNRAS, 464, 385

Bon, E., Jovanović, P., Marziani, P., et al. 2012, ApJ, 759, 118

Bon, E., Zucker, S., Netzer, H., et al. 2016, ApJS, 225, 29

Boroson, T. A., \& Green, R. F. 1992, ApJS, 80, 109

Cackett, E. M., \& Horne, K. 2006, MNRAS, 365, 1180

Calvani, M., Marziani, P., Bachev, R., et al. 2004, Mem. Soc. Astron. It. Suppl., 5,223

Carswell, R. F., \& Smith, M. G. 1978, MNRAS, 185, 381

Chen, K., Halpern, J. P., \& Filippenko, A. V. 1989, ApJ, 339, 742

Clavel, J., Boksenberg, A., Bromage, G. E., et al. 1990, MNRAS, 246, 668

Crenshaw, D. M., Rodriguez-Pascual, P. M., Penton, S. V., et al. 1996, ApJ, 470, 322

Croom, S. M., Rhook, K., Corbett, E. A., et al. 2002, MNRAS, 337, 275

Dietrich, M., Hamann, F., Shields, J. C., et al. 2002, ApJ, 581, 912

Dong, X., Wang, T., Wang, J., et al. 2008, MNRAS, 383, 581

Dong, X., Wang, J., Wang, T., et al. 2009, in The Starburst-AGN Connection, eds. W. Wang, Z. Yang, Z. Luo, \& Z. Chen, ASP Conf. Ser., 408, 83

Ferland, G. J., Korista, K. T., Verner, D. A., et al. 1998, PASP, 110, 761

Ferland, G. J., Porter, R. L., van Hoof, P. A. M., et al. 2013, Rev. Mex. Astron. Astrofis., 49, 137

Gilbert, K. M., \& Peterson, B. M. 2003, ApJ, 587, 123

Goad, M. R., \& Korista, K. T. 2014, MNRAS, 444, 43

Goad, M. R., \& Korista, K. T. 2015, MNRAS, 453, 3662

Goad, M. R., Korista, K. T., \& Knigge, C. 2004, MNRAS, 352, 277

Green, P. J. 1996, ApJ, 467, 61

Green, P. J. 1998, ApJ, 498, 170

Green, P. J., Forster, K., \& Kuraszkiewicz, J. 2001, ApJ, 556, 727

Greene, J. E., \& Ho, L. C. 2005, ApJ, 630, 122

Ilić, D., Popović, L. Č., Ciroi, S., \& Rafanelli, P. 2008, Rev. Mex. Astron. Astrofis., 32, 102

Ilić, D., Popović, L. V. C., Shapovalova, A. I., León-Tavares, J., \& Chavushyan, V. H. 2010, in Accretion and Ejection in AGN: a Global View, eds L. Maraschi, G. Ghisellini, R. Della Ceca, \& F. Tavecchio, ASP Conf. Ser., 427, 110

Ilić, D., Popović, L. Č., La Mura, G., Ciroi, S., \& Rafanelli, P. 2012, A\&A, 543, A 142

Ilić, D., Popović, L. Č., Shapovalova, A. I., et al. 2015, JApA, 36, 433

Jiang, P., Wang, J. X., \& Wang, T. G. 2006, ApJ, 644, 725

Jiang, P., Wang, J.-X., \& Wang, T.-G. 2007, in The Central Engine of Active Galactic Nuclei, eds. L. C. Ho, \& J.-W. Wang, ASP Conf. Ser., 373, 143

Jovanović, P., Popović, L. Č., Stalevski, M., \& Shapovalova, A. I. 2010, ApJ, 718,168

Kara, E., Fabian, A. C., Cackett, E. M., et al. 2013, MNRAS, 434, 1129

Kaspi, S., Maoz, D., Netzer, H., et al. 1996, ApJ, 470, 336

Kinney, A. L., Rivolo, A. R., \& Koratkar, A. P. 1990, ApJ, 357, 338

Kong, M.-Z., Wu, X.-B., Wang, R., Liu, F. K., \& Han, J. L. 2006, A\&A, 456, 473

Korista, K. 1999, in Quasars and Cosmology, eds. G. Ferland, \& J. Baldwin, ASP Conf. Ser., 162, 429
Korista, K. T., \& Goad, M. R. 2004, ApJ, 606, 749

Korista, K., Baldwin, J., Ferland, G., \& Verner, D. 1997, ApJS, 108, 401 Korista, K., Baldwin, J., \& Ferland, G. 1998, ApJ, 507, 24

Kovačević, J., Popović, L. Č., \& Dimitrijević, M. S. 2010, ApJS, 189, 15

La Mura, G., Popović, L. Č., Ciroi, S., Rafanelli, P., \& Ilić, D. 2007, ApJ, 671, 104

Leahy, J. P., \& Perley, R. A. 1995, MNRAS, 277, 1097

Li, Y.-R., Wang, J.-M., Ho, L. C., et al. 2016, ApJ, 822, 4

Maoz, D., Netzer, H., Mazeh, T., et al. 1991, ApJ, 367, 493

Marziani, P., Dultzin, D., \& Sulentic, J. W. 2008, Rev. Mex. Astron. Astrofis., 32, 103

Mushotzky, R., \& Ferland, G. J. 1984, ApJ, 278, 558

Netzer, H. 1985, MNRAS, 216, 63

Netzer, H., Laor, A., \& Gondhalekar, P. M. 1992, MNRAS, 254, 15

Netzer, H., Brotherton, M. S., Wills, B. J., et al. 1995, ApJ, 448, 27

Osmer, P. S., \& Shields, J. C. 1999, in Quasars and Cosmology, eds. G. Ferland, \& J. Baldwin, ASP Conf Ser., 162, 235

Osterbrock, D. E., \& Ferland, G. J. 2006, Astrophysics of gaseous nebulae and active galactic nuclei (Sausalito, CA: University Science Books)

Penston, M. V., Clavel, J., Snijders, M. A. J., Boksenberg, A., \& Fosbury, R. A. E. 1979, MNRAS, 189, 45

Peterson, B. M., \& Cota, S. A. 1988, ApJ, 330, 111

Peterson, B. M., Pogge, R. W., Wanders, I., Smith, S. M., \& Romanishin, W. 1995, PASP, 107, 579

Peterson, B. M., Berlind, P., Bertram, R., et al. 2002, ApJ, 581, 197

Pogge, R. W., \& Peterson, B. M. 1992, AJ, 103, 1084

Popović, L. C. 2003, ApJ, 599, 140; Erratum: 2006, ApJ, 650, 1217

Popović, L. C. 2012, New Astron. Rev., 56, 74

Popović, L. Č., \& Kovačević, J. 2011, ApJ, 738, 68

Popović, L. Č., Shapovalova, A. I., Chavushyan, V. H., et al. 2008, PASJ, 60, 1

Popović, L. Č., Shapovalova, A. I., Ilić, D., et al. 2011, A\&A, 528, A130

Popović, L. Č., Shapovalova, A. I., Ilić, D., et al. 2014, A\&A, 572, A66

Ricci, C., Paltani, S., Awaki, H., et al. 2013, A\&A, 553, A29

Robinson, A., Young, S., Axon, D. J., Kharb, P., \& Smith, J. E. 2010, ApJ, 717 L122

Runnoe, J. C., Cales, S., Ruan, J. J., et al. 2016, MNRAS, 455, 1691

Sergeev, S. G., Pronik, V. I., Peterson, B. M., Sergeeva, E. A., \& Zheng, W. 2002, ApJ, 576, 660

Shang, Z., Wills, B. J., Robinson, E. L., et al. 2003, ApJ, 586, 52

Shapovalova, A. I., Doroshenko, V. T., Bochkarev, N. G., et al. 2004, A\&A, 422, 925

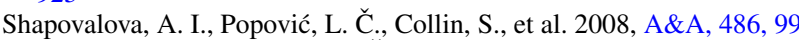

Shapovalova, A. I., Popović, L. Č., Burenkov, A. N., et al. 2010a, A\&A, 517, A42

Shapovalova, A. I., Popović, L. Č., Burenkov, A. N., et al. 2010b, A\&A, 509, A106

Shapovalova, A. I., Popović, L. Č., Burenkov, A. N., et al. 2012, ApJS, 202, 10

Shapovalova, A. I., Popović, L. Č., Burenkov, A. N., et al. 2013, A\&A, 559, A10

Shapovalova, A. I., Popović, L. Č., Chavushyan, V. H., et al. 2016, ApJS, 222, 25

Shemmer, O., \& Lieber, S. 2015, ApJ, 805, 124

Shemmer, O., Romano, P., Bertram, R., et al. 2001, ApJ, 561, 162

Shen, Y., \& Ho, L. C. 2014, Nature, 513, 210

Shen, Y., Richards, G. T., Strauss, M. A., et al. 2011, ApJS, 194, 45

Shields, J. C. 2007, in The Central Engine of Active Galactic Nuclei, eds. L. C. Ho, \& J.-W. Wang, ASP Conf. Ser., 373, 355

Shu, X. W., Wang, J. X., Yaqoob, T., Jiang, P., \& Zhou, Y. Y. 2012, ApJ, 744, L2

Sulentic, J. W., Zwitter, T., Marziani, P., \& Dultzin-Hacyan, D. 2000, ApJ, 536, L5

Sulentic, J. W., Marziani, P., \& Zamfir, S. 2009, New Astron. Rev., 53, 198

Wamsteker, W., \& Colina, L. 1986, ApJ, 311, 617

Wandel, A. 1999a, ApJ, 527, 657

Wandel, A. 1999b, in Quasars and Cosmology, eds. G. Ferland, \& J. Baldwin, ASP Conf. Ser., 162, 335

Warner, C., Hamann, F., \& Dietrich, M. 2003, ApJ, 596, 72

Xu, Y., Bian, W.-H., Yuan, Q.-R., \& Huang, K.-L. 2008, MNRAS, 389, 1703

Yang, J., \& Zhou, B. 2015, PASJ, 67, 124

Zamorani, G., Marano, B., Mignoli, M., Zitelli, V., \& Boyle, B. J. 1992, MNRAS, 256, 238

Zheng, W., \& Malkan, M. A. 1993, ApJ, 415, 517

Zheng, W., Fang, L.-Z., \& Binette, L. 1992, ApJ, 392, 74

Zhou, H., Wang, T., Yuan, W., et al. 2006, ApJS, 166, 128 